To the Editors:

\title{
Outcome of hepatic resection: First five-year experience in elderly and younger patients
}

\author{
B K S Bulathsinhala ${ }^{1}$, M S B Tillekaratne ${ }^{2}$, M B Gunetilleke ${ }^{2}$, M A Niriella ${ }^{3}$, D G A Wijegunawardena ${ }^{1}$, \\ R C Siriwardana ${ }^{2}$
}

Ceylon Medical Journal 2018; 63: 43-45

DOI: http://doi.org/10.4038/cmj.v63i1.8617

(Index words: Hepatic resection, Perioperative outcome, Perioperative mortality, elderly, hepatocellular carcinoma)

\section{Introduction}

Hepatic resection offers the best curative treatment for most surgical liver diseases [1,2]. However, liver surgery is demanding. It requires meticulous technique, careful planning, supportive care and infrastructure. Over the years advances in liver surgery has made it possible to carry it out safely even in extreme cases [3].

Hepatobiliary surgery is rapidly developing in Sri Lanka. Increasing number of patients are referred with surgical liver disease. Managing these patients are challenging in newly established centers. This study analysed the outcome of the first 150 patients who underwent liver resection at the professorial surgical unit of the North Colombo Teaching Hospital, Ragama from 2012 to 2017. Patients with hepatocellular carcinoma, cholangiocarcinoma, colorectal liver metastases, noncolorectal liver metastases and benign liver lesions who underwent curative resection were included. Emergency hepatic resection following trauma were excluded. All data were collected prospectively. Demographic, clinical, laboratory data and comorbidities were recorded. Outcome measures analysed were, intraoperative details such as total operative time, hepatic transection time and intraoperative blood loss. Post-operative outcomes were duration of intensive care unit (ICU) stay, duration of hospital stay, major and minor postoperative complications and mortality rate. Patients were divided in to two groups for comparison. Elderly group was defined as patients aged 65 years or more $(n=49)$ and younger group was defined as patients below 65 years $(n=101)$.
Diagnosis of liver pathology was primarily made on cross sectional imaging. All images were reported by two radiologists. Liver biopsy was performed in selected cases, where diagnoses were inconclusive. All patients were discussed in a dedicated multidisciplinary meeting including a hepatobiliary surgeon, hepatologist, oncologist and two specialist radiologists.

Selection for surgery was based on patient's general condition, performance status, liver status, location of liver lesion, functional liver reserves and patient's consent. Advanced age itself was not a limiting factor for surgery. Patients who were not selected for surgery were offered ablation therapy, transarterial chemotherapy (TACE) or palliative care accordingly.

Hepatic resections were done according to standardized anaesthesia protocols and advanced intraoperative monitoring. Harmonic scalpel and Cavitron Ultrasonic Surgical Aspirator (CUSA) were primarily used for hepatic transections. CVP was maintained close to zero. Pringles maneuver was used selectively. Patients were closely followed up at a dedicated clinic after the surgery.

Data was analyzed using SPSS software version 17. Data were presented as mean with standard deviation (SD), median with interquartile range (IQR) and frequencies with percentages $(\%)$. The differences between groups were evaluated using Pearson's Chi-square test, Mann Whitney U, Kruskal-Wallis test as appropriate. A P value of less than 0.05 was considered statistically significant.

${ }^{1}$ Professorial Surgical Unit, North Colombo Teaching Hospital, Ragama, ${ }^{2}$ Department of Surgery, Faculty of Medicine, University of Kelaniya, Ragama, ${ }^{3}$ Department of Medicine, Faculty of Medicine, University of Kelaniya, Ragama, Sri Lanka.

Correspondence: RCS, e-mail: <rohansiriwardana@yahoo.com>. Received 09 January 2018 and revised version accepted 16 January 2018.

This is an open-access article distributed under the terms of the Creative Commons Attribution License, which permits unrestricted use, distribution, and reproduction in any medium, provided the original author and source are credited. 
Out of 150 patients who underwent hepatic resections, elderly group consisted of 49 patients. Of them $71.4 \%$ were males, median age was 69 years (range $65-79$ ). There were 101 patients in the younger group, $59.4 \%$ were males, median age was 54 years (range 12-64].

Preoperative base line investigations and comorbidities were compared between the two groups. Serum creatinine $(p=0.034)$, renal disease $(p=0.011)$ and respiratory diseases $(p=0.015)$ were significantly higher in the elderly group.

Seventy five resections $(50 \%)$ were done for hepatocellular carcinoma, eight $(5.3 \%)$ were for cholangiocarcinoma, twenty five $(16.6 \%)$ for colorectal liver metastases, twelve ( $8 \%$ ) for non-colorectal liver metastases and thirty (20\%) for benign liver lesions.

Hundred and twenty five $(83.3 \%)$ of the hepatic resections were done as open procedures. Others were laparoscopic resections. One case was converted to open.

Right hepatectomy was the commonest type of resection $(n=51 ; 34.6 \%)$, followed by wedge resection $(21.3 \%)$ and monosegmentectomy $(14.6 \%)$. The types of resection in the two groups were similar.

Median operating time in the elderly was 240 minutes (range 70-600) compared to 285 minutes (range 24-630) in the younger group $(p=0.55)$. Hepatic transection time in the elderly group was 90 minutes (range 30-240) and in the younger group 77.5 minutes (range 30-210) $(p=0.81$ ). Median intraoperative blood loss was $350 \mathrm{ml}$ (range 20-2000) in the elderly compared to $300 \mathrm{ml}$ (range 10-2800) in the younger group $(P=0.696)$. Median hospital stay was 8 minutes in the elderly group and 7 days in the younger group ( $p=0.229$ ). Median ICU stay was 2 days in both groups (Table 1).

Postoperative complications were categorized as major and minor. Of the elderly group $7.2 \%$ had major complications and $24.2 \%$ had minor complications. In the younger groups $5.5 \%$ had major complications, and $17.16 \%$ had minor complications (Table 1).

Table 1. Comparison of pre-operative MELD score, percentages of comorbidities, types of hepatic resections, intraoperative and perioperative outcome and mortality between elderly and younger groups

\begin{tabular}{|c|c|c|c|}
\hline & Elderly $(n=49)$ & Younger $(n=101)$ & $p$ Value \\
\hline \multicolumn{4}{|l|}{ Preoperative MELD score } \\
\hline MELD score & 8.0 & 9.0 & 0.938 \\
\hline \multicolumn{4}{|l|}{ Comorbidities } \\
\hline Diabetes mellitus & $38.8 \%(n=19)$ & $18.5 \%(n=19)$ & 0.773 \\
\hline Hypertension & $7.2 \%$ & $8.7 \%$ & 0.673 \\
\hline Ischemic heart disease & $2.4 \%$ & $5.4 \%$ & 0.721 \\
\hline Ischemic heart disease and hypertension & $4.8 \%$ & $5.4 \%$ & \\
\hline Renal disease & - & $1.1 \%$ & 0.011 \\
\hline Respiratory disease & $2.4 \%$ & $2.2 \%$ & 0.015 \\
\hline \multicolumn{4}{|l|}{ Type of hepatic resection } \\
\hline Major resection & 26 & 50 & \\
\hline Minor resection & 23 & 51 & \\
\hline \multicolumn{4}{|c|}{ Intraoperative and perioperative outcome } \\
\hline Total operating time (minutes) & $240(70-600)$ & $285(24-630)$ & 0.55 \\
\hline Hepatic transection time (minutes) & $90(30-240)$ & $77.5(30-210)$ & 0.81 \\
\hline Intraoperative blood loss $(\mathrm{ml})$ & $350(20-2000)$ & $300(10-28000$ & 0.696 \\
\hline Total hospital stay (days) & $8(2-31)$ & $7(3-30)$ & 0.229 \\
\hline Total ICU stay (days) & $2(1-5)$ & $2(1-10)$ & 0.509 \\
\hline \multicolumn{4}{|c|}{ Perioperative complications and mortality } \\
\hline Major complications & $7.2 \%$ & $5.5 \%$ & \\
\hline Minor complications & $24.2 \%$ & $17.16 \%$ & \\
\hline No complications & $68.6 \%$ & $77.34 \%$ & \\
\hline Perioperative mortality & $6.12 \%$ & $3.26 \%$ & \\
\hline
\end{tabular}

INR - International normalized ratio; MELD score - Model for End-Stage Liver Disease score 
Studies have reported varied complication rates following hepatic resections. Overall morbidity of $22.5 \%$ was reported in a study which analyzed 1500 consecutive cases over 20 years. Perioperative mortality ranges from $2-5 \%$ [4]. Interestingly some studies report higher operative mortality in younger $(9.6 \%)$ patients compared to elderly (3.1\%) patients [2]. In our series perioperative mortality in the elderly group was $6.12 \%$ and $3.26 \%$ in younger group. The difference was not statistically significance $(p=0.47)$. These rates are comparable to those in other studies.

Safety of hepatic resection has improved dramatically during the last few decades [1,2]. Careful preoperative evaluation, assessment of functional liver reserves, advancement of surgical techniques and perioperative management has shown a better outcome in surgical candidates [1]. High volume hepatobiliary centers embark on more advanced hepatic resections with experience and show notable improvement in peri-operative outcomes [5].

Even though this clinical service was started recently, a well organized system has resulted in outcomes which are comparable with other centers, with an overall mortality of $6.12 \%$ in the elderly and $3.26 \%$ in the younger group. Though laparoscopic resection may have been tolerated well we are still in the early stage of the learning curve.

In conclusion, in a recently started clinical service multidisciplinary input, careful patient selection, advanced preoperative optimization, improved anaesthesia, surgical techniques and meticulous perioperative care in a high volume center has achieved comparable outcome in elderly patients after hepatic resection.

\section{Acknowledgement}

We thank Professor Aloka Pathirana, Dr. Shantha Hettiarachchi, Dr. Lakmali Paranahewa and Dr. Dehan Gunasekara members of hepatobiliary multidisciplinary team.

\section{Conflicts of interest}

There are no conflicts of interest.

\section{References}

1. Nanashima A, Abo T, Nonaka T, et al. Prognosis of patients with hepatocellular carcinoma after hepatic resection: Are elderly patients suitable for surgery? J Surg Oncol 2011; 104(3): 284-91.

2. Ferrero A, Viganò L, Polastri R, et al. Hepatectomy as treatment of choice for hepatocellular carcinoma in elderly cirrhotic patients. World J Surg 2005; 29(9): 1101-5.

3. Mann CD, Neal CP, Pattenden CJ, et al. Major resection of hepatic colorectal liver metastases in elderly patients An aggressive approach is justified. Eur J Surg Oncol 2008; 34(4): 428-32.

4. Cescon M, Vetrone G, Grazi GL, et al. Trends in perioperative outcome after hepatic resection: analysis of 1500 consecutive unselected cases over 20 years. Ann Surg 2009; 249(6): 995-1002.

5. Dimick JB, Wainess RM, Cowan JA, Upchurch GR, Knol JA, Colletti LM. National trends in the use and outcomes of hepatic resection. J Am Coll Surg 2004; 199(1): 31-8. 